Pontifícia Universidade C $_{\text {atólica }}$

Thiago Marques Toledo

\title{
Visualizando Campos Vetoriais em Superfícies
}

Dissertação apresentada como requisito parcial para obtenção do grau de Mestre pelo Programa de Pós-graduação em Informática do Departamento de Informática da PUC-Rio

Orientador: Prof. Waldemar Celes Filho 


\title{
Thiago Marques Toledo
}

\section{Visualizando Campos Vetoriais em Superfícies}

Dissertação apresentada como requisito parcial para obtenção do grau de Mestre pelo Programa de Pós-graduação em Informática do Departamento de Informática do Centro Técnico Científico da PUC-Rio. Aprovada pela comissão examinadora abaixo assinada.

\author{
Prof. Waldemar Celes Filho \\ Orientador \\ Departamento de Informática — PUC-Rio \\ Prof. Marcelo Gattass \\ Departamento de Informática — PUC-Rio \\ Prof. Hélio Côrtes Vieira Lopes \\ Departamento de Informática — PUC-Rio
}

Prof. Luiz Henrique de Figueiredo Instituto Nacional de Matemática Pura e Aplicada

Prof. José Eugenio Leal

Coordenador Setorial do Centro Técnico Científico - PUC-Rio 
Todos os direitos reservados. Proibida a reprodução total ou parcial do trabalho sem autorização da universidade, do autor e do orientador.

Thiago Marques Toledo

Graduou-se em Sistemas de Informação na Pontifícia Universidade Católica do Rio de Janeiro, com ênfase em computação gráfica. Trabalhou no Grupo de Tecnologia em Computação Gráfica da PUC-Rio (Tecgraf) como pesquisador e desenvolvedor em projetos relacionados à indústria de petróleo.

Ficha Catalográfica

Toledo, Thiago Marques

Visualizando Campos Vetoriais em Superfícies / Thiago Marques Toledo; orientador: Waldemar Celes Filho. - Rio de Janeiro : PUC-Rio, Departamento de Informática, 2012.

v., 51 f: il. ; $29,7 \mathrm{~cm}$

1. Dissertação (Mestrado em Informática) - Pontifícia Universidade Católica do Rio de Janeiro, Departamento de Informática.

Inclui referências bibliográficas.

1. Informática - Tese. 2. Convolução por Integral de Linha. 3. Campos Vetoriais. 4. Visualização Científica. 5. Computação Gráfica. I. Filho, Waldemar Celes. II. Pontifícia Universidade Católica do Rio de Janeiro. Departamento de Informática. III. Título. 


\section{Agradecimentos}

A meus pais Nei e Mariangela e a toda minha família, pelo apoio incondicional. A minha avó Dulce Toledo, em especial, por todos os anos de suporte e torcida, dos quais jamais esquecerei.

A meu orientador e demais professores do Departamento de Informática pelo apoio fundamental à realização deste trabalho. A meus colegas do laboratório Tecgraf, sempre pacientes e dispostos a ajudar.

Ao $\mathrm{CNPq}$ e à PUC-Rio, pelos auxílios concedidos, que viabilizaram este trabalho. 


\section{Resumo}

Toledo, Thiago Marques; Filho, Waldemar Celes. Visualizando

Campos Vetoriais em Superfícies. Rio de Janeiro, 2012. 51p. Dissertação de Mestrado - Departamento de Informática, Pontifícia Universidade Católica do Rio de Janeiro.

Campos vetoriais são resultados comuns em simuladores físicos. Simulações em modelos de reservatórios de petróleo podem nos fornecer, por exemplo, dados relativos ao fluxo de óleo, água e gás. Para um melhor entendimento de tais dados, entretanto, é interessante o uso de uma técnica de visualização que permita a identificação de características locais e tendências globais no campo. Este trabalho propõe uma técnica para visualização de campos vetoriais 3D baseada em GPU que utiliza o algoritmo de convolução de integral de linha (LIC) em 2D para a visualização da componente tangencial à superfície projetada no espaço da tela. Dados relativos à magnitude e componente normal são apresentados através de uma escala de cores bidimensional. Para fixar a imagem resultante do LIC no modelo é proposto um esquema simples baseado em coordenadas de texturas aleatórias, eliminando a necessidade de textura sólida 3D para armazenar o ruído branco. Filtros para animação da imagem de LIC foram adaptados para permitir velocidade variável de acordo com a magnitude do campo. Para melhoria da imagem final, o algoritmo de LIC é aplicado em duas passadas e o resultado é submetido a um filtro de passa-alta. O framework desenvolvido como parte do trabalho foi explorado no contexto da visualização de fluxos em modelos de reservatório de petróleo e de gradientes de altura em terrenos. No caso específico de reservatórios, é proposta uma variação da técnica que permite visualização simultânea de fluxos de óleo, gás e água.

\section{Palavras-chave}

Convolução por Integral de Linha; Campos Vetoriais; Visualização Científica ; Computação Gráfica. 


\section{Abstract}

Toledo, Thiago Marques; Filho, Waldemar Celes (advisor). Visualizing Vector Fields over Surfaces. Rio de Janeiro, 2012. 51p. MSc. Dissertation - Departamento de Informática, Pontifícia Universidade Católica do Rio de Janeiro.

Vector fields are common results of physics simulators. Simulations over black-oil reservoirs, for instance, can generate oil, water and gas flow data. For a better understanding of such data, however, it's interesting to use a visualization technique that allows a better identification of local characteristics and global tendencies of the field. This work proposes a technique for visualization of 3D vector fields that is GPU-based and uses the 2D line integral convolution (LIC) algorithm to visualize the component tangential to the surface projected on screen space. Data related to magnitude and normal component are presented through a 2-dimensional color scale. A simple scheme based on randomly generated texture coordinates is proposed to fixate the resulting LIC image to the model, avoiding flickering during model manipulation and eliminating the need for a solid 3D texture noise. For animation, we adjust the use of filters to ensure that the animation speed varies in accordance to the field magnitude. To enhance the final image, the LIC algorithm is applied in two passes and the result is put through a high-pass filter. The framework developed as part of this work has been applied in the context of visualizing flow in black-oil reservoir models and height gradients in terrains. In the specific case of reservoirs, a variation from the main technique is proposed to allow simultaneous visualization of oil, gas and water flows.

\section{Keywords}

Line Integral Convolution; Vector Fields; Scientific Visualization; Computer Graphics. 


\section{Sumário}

1 Introdução 12

2 Trabalhos Relacionados $\quad 14$

2.1 Convolução por Integral de Linha 14

$\begin{array}{ll}2.2 \text { LIC com Melhorias } & 18\end{array}$

2.3 Visualização de Fluxo Baseada em Imagens 20

2.4 Outros Trabalhos 21

3 Técnica Proposta $\quad 22$

3.1 LIC Revisitado $\quad 22$

3.2 Trabalhando no Espaço da Tela 24

$\begin{array}{ll}3.3 & \text { Problemas e Soluções } \\ 3.4 & 26\end{array}$

3.4 Técnica Final 32

4 Implementação $\quad 36$

4.1 Visão Geral da Biblioteca 36

4.2 Instanciando um Campo 36

$\begin{array}{lll}4.3 & \text { A Biblioteca } & 37\end{array}$

5 Aplicações $\quad 39$

5.1 Geresim 39

5.2 Georisco 42

$\begin{array}{lll}6 & \text { Conclusão } & 49\end{array}$

$7 \quad$ Referências Bibliográficas $\quad 50$ 


\section{Lista de figuras}

1.1 Visualização de campos vetoriais em 3D com linhas de fluxo. 12

2.1 Imagem típica do algoritmo de convolução por integral de linha. 14

2.2 Linha de fluxo avaliada para um pixel no algoritmo de LIC. Imagem extraída do artigo original (2).

2.3 A partir da linha de fluxo e da imagem de ruído, é obtida a cor final por convolução. Imagem extraída do artigo original (2). 16

$\begin{array}{lll}2.4 & \text { Diferentes imagens de ruído aplicadas ao LIC. } & 18\end{array}$

2.5 Comparação entre LIC convencional e LIC duplo. 19

2.6 Filtro de passa-alta aplicado ao resultado de duas passadas de LIC. 20

3.1 Projeção de um vetor do campo no espaço da tela. 25

3.2 Aplicação de escala de cores (Branco-Verde) representando magnitude.

3.3 Aplicação de escala de cores (Azul-Branco-Vermelho) representando componente normal.

3.4 Escala bidimensional de cores para representar magnitude e componente normal.

3.5 Aplicação de paleta de cores representando componente normal e magnitude.

3.6 Campo vetorial homogêneo representado sobre um toro.

3.7 Campo homogêneo $\hat{f}=(0,1,0)$ sobre toro com e sem alinhamento, respectivamente.

3.8 Campo homogêneo $\hat{f}=(0, \sqrt{2},-\sqrt{2})$ sobre toro com e sem alinhamento, respectivamente.

3.9 Campos homogêneos $\hat{f}=(0,1,0)$ e $\hat{f}=(0, \sqrt{2},-\sqrt{2})$, respectivamente, alinhados e com paleta de cores.

3.10 Etapas de pré-processamento e projeção.

3.11 Etapa de Convolução por Integral de Linha.

3.12 Etapa de Aplicação do Filtro Passa-Alta.

5.1 Geresim: Visualização de modelo de reservatório em 3D.

5.2 Geresim: Visualização de fluxo de óleo em mapa.

5.3 Geresim: Visualização de uma camada do modelo.

5.4 Geresim: Visualização de uma seção do modelo.

5.5 Geresim: Visualização de múltiplas camadas.

5.6 Geresim: Visualização de corte entre camadas e seções em um poço produtor.

5.7 Geresim: Visualização trifásica.

5.8 Geresim: Visualização de LIC Trifásico.

5.9 Geresim: Visualização de LIC Trifásico (Área de alta saturação de gás e óleo).

5.10 Georisco: Imagem típica do sistema.

5.11 Georisco: Visão geral do gradiente de altura.

5.12 Georisco: Aproximação de uma região de alta declividade. 
5.13 Georisco: Gradiente apresentado com visão de topo sobre o terreno achatado. 


\section{Lista de tabelas}

3.1 Conteúdo das texturas geradas na etapa de projeção. 34

4.1 Código em GLSL para projeção de um vetor do modelo. 38 
An alleged scientific discovery has no merit unless it can be explained to a barmaid.

Ernest Rutherford 